Article

\title{
Bird-Wing Optical-Reflector Design with Photocatalyst for Low-Glare Mosquito Trapping System with Light-Emitting Diodes
}

\author{
Wei-Hsiung Tseng ${ }^{1}$, Diana Juan ${ }^{2}$, Wei-Cheng Hsiao ${ }^{2}$, Yi Chian Chen ${ }^{1}$, Cheng-Han Chan ${ }^{3}$, \\ Hsin-Yi Ma ${ }^{4}$ and Hsiao-Yi Lee ${ }^{1,5, *}$ \\ 1 Department of Electrical Engineering, National Kaohsiung University of Science and Technology, \\ Kaohsiung City 807, Taiwan; tly885@gmail.com (W.-H.T.); al11062000@gmail.com (Y.C.C.) \\ 2 Yuan General Hospital, Kaohsiung City 802, Taiwan; dianajuan@yuanhosp.com.tw (D.J.); \\ cheng_2034@yahoo.com.tw (W.-C.H.) \\ 3 Department of Aviation and Communication Electronics, Air Force Institute of Technology, \\ Kaohsiung City 820, Taiwan; errmatlab@gmail.com \\ 4 Department of Industrial Engineering and Management, Minghsin University of Science and Technology, \\ Hsinchu County 30401, Taiwan; hsma@must.edu.tw \\ 5 Department of Graduate Institute of Clinical Medicine, Kaohsiung Medical University, \\ Kaohsiung City 807, Taiwan \\ * Correspondence: leehy@nkust.edu.tw
}

Received: 11 February 2019; Accepted: 5 March 2019; Published: 7 March 2019

\begin{abstract}
Dengue fever is the most serious vector-borne disease in Asia. There are still no dengue vaccines or therapeutic drugs, and vector-mosquito control is the main prevention and treatment method. The prevention and control of dengue-fever vector mosquitoes in Taiwan is still dominated by larval control. However, the removal of the source of mosquito-borne diseases has not been fully implemented, and the removal process of vector-borne mosquitoes cannot keep up with their breeding rate. In addition, chemical agents used in the elimination of pathogenic mosquitoes may cause mosquito resistance and environmental pollution. Therefore, it is important to develop new prevention and control technologies. This study is dedicated to the development of a mosquito trapping optical system with high efficiency and low glare that is safe for humans. The system is mainly equipped with ultraviolet light-emitting diodes (UV-LED), a freeform-surfaced optical reflector, and a photocatalyst. The reflector can lead light downward for the protection of user eyes, and expand the range of trapping mosquitoes to $225 \pi \mathrm{m}^{2}$. Based on practical experiments, captured mosquitoes increased by about $450 \%$ through the proposed system compared to conventional traps using UV LED. In addition, the proposed system is shown to be $45 \%$ more enhanced in trapping capability after a photocatalyst (titanium dioxide) coating is applied to its reflector.
\end{abstract}

Keywords: light-emitting diodes (LED); low-glare mosquito trapping system; optical reflector with free-form surfaces; photocatalyst coating (titanium dioxide)

\section{Introduction}

Mosquitoes are the vector of many diseases, especially in environments with high temperatures and humidity, which are more suitable for the growth and reproduction of mosquitoes and diseases transmitted by them, making them more serious in this kind of environment. According to the statistics from the World Health Organization, about one million people on average die each year from infectious diseases spread by mosquitoes [1]. For instance, filariasis and the West Nile virus are transmitted by Culex quinquefasciatus [2], the Japanese encephalitis virus (J.E.V.) is transmitted by Culex annulus, 
and dengue fever and the Zika virus [3,4] are transmitted by Aedes aegypti and/or Aedes albopictus. Therefore, methods effectively reducing the number of mosquitoes and preventing them from biting human skin are a concerning issue to everyone.

The prevention and control of dengue-fever vector mosquitoes in Taiwan is still dominated by larval control [5]. However, the removal of the source of mosquito-borne diseases has not been fully implemented, and the removal of vector-borne mosquitoes cannot keep up with their breeding rate. In addition, chemical agents used in the elimination of pathogenic mosquitoes may cause mosquito resistance and environmental pollution.

Mosquitoes are highly sensitive to optical intensity, direction, wavelength, and contrast ratio [6,7]. Studies of sensitivity of mosquito eye to ultraviolet (UV) rays or short-wavelength visible light have been conducted [8-10]. According to our investigation, current commercialized mosquito trappers are not quite satisfactory with customers with regard to their trapping capability and due to their uncomfortable glare. As a result, a low-glare mosquito trapping system with high trapping capabilities is proposed in this study. The proposed system is equipped with an optimized optical reflector with a freeform surface coated with a titanium dioxide as a photocatalyst. The reflector can transform the LED source flux as appropriate light distribution in space to attract mosquitoes more easily. The photocatalyst coating (titanium dioxide) was adopted on the reflector to increase carbon dioxide concentration and simulate human exhalation like in the enviroment, which is demonstrated to increase the power of the proposed device in trapping mosquitoes.

\section{Principles and Methods}

It is known that the visible spectrum of most insects falls between 300 and $700 \mathrm{~nm}$ (Briscoe and Chittka, 2001) [11]. In this spectrum, ultraviolet, blue, green and other wavelength ranges are more likely to attract mosquitoes. A study by Muir et al. [12] in 1992 pointed out that Aedes albopictus is better attracted at wavelengths of between about 340 and $520 \mathrm{~nm}$. A study by Burkett and Butler in 2005 pointed out that both Aedes aegypti and Aedes albopictus have different degrees of attraction in a wavelength range from 350 to $700 \mathrm{~nm}$ [13]. In the study, the physical properties of attractants, including specific light wavelengths, and supplemented with a photocatalyst coating of titanium dioxide on mosquito traps, were used to increase carbon dioxide concentration to simulate human living environments and enhance the lure of dengue-vector mosquitoes.

Ultraviolet light is harmful to the human eye, but conventional mosquito trappers, as shown in Figure 1, arranges the UV light source in their inner space to preventing direct contact with human eyes. In consequence, commercial mosquito trappers have the problems of low light intensity and a strong glare to human eyes. The proposed mosquito trapping device utilizes ultraviolet LED as its light source to attract mosquitoes, and an air-inhalation flow system to trap and kill them, as shown in Figure 2. The airflow vortex inhalation system was designed to trap mosquitoes, preventing them from escaping. The airflow, generated by a fan hidden in the inner space of the mosquito trapper, targets the mosquito habit to move with the airflow, guiding the wandering mosquitoes to gather at and get sucked into the mosquito box in the reflective cavity. The continuous airflow goes into the mosquito-collection area, causing the mosquito to die due to air-drying and dehydration from the inhalation airflow. The safe and hygienic mosquito-collection area can reduce the spread of bacteria carried by mosquitoes, which is superior to the electric shock-type mosquito trap system. Mosquitoes have phototaxis characteristics, attracting surrounding mosquitoes by light trapping. By entering the reflective cavity area, mosquitoes are inadvertently inhaled into the mosquito-collection area with the airflow until they are killed.

A freeform-surfaced optical reflector was designed to generate effective light spatial distribution and provide enough light-soure excitation to the photocatalyst coating for producing $\mathrm{CO}_{2}$, creating a simulation of human exhalation and increasing attractiveness to mosquitoes. The freeform-surfaced optical reflector is like a bird wing. Each wing has three segmented reflecting portions with length $D_{i}$ and tilt angle $\theta_{\mathrm{i}}$, as shown in Figure 3. A UV light source is located under the center of the bird-wing 
reflector. The height from the source to the center and the end of the reflector is $\mathrm{H}_{1}$ and $\mathrm{H}_{2}$, respectively. In the design processes, $\mathrm{Hi}, \mathrm{Di}$, and $\theta_{\mathrm{i}}$ are used as variables to accomplish the targeted reflected light distribution. The reflector is optimized to concentrate light downward and prevent it from meeting users' eyes, enhancing light intensity and expanding its reach much further than conventional reflectors. The reflector-design workflow with the application of $\mathrm{TiO}_{2}$ to the LED mosquito trapping system is shown in Figure 4.

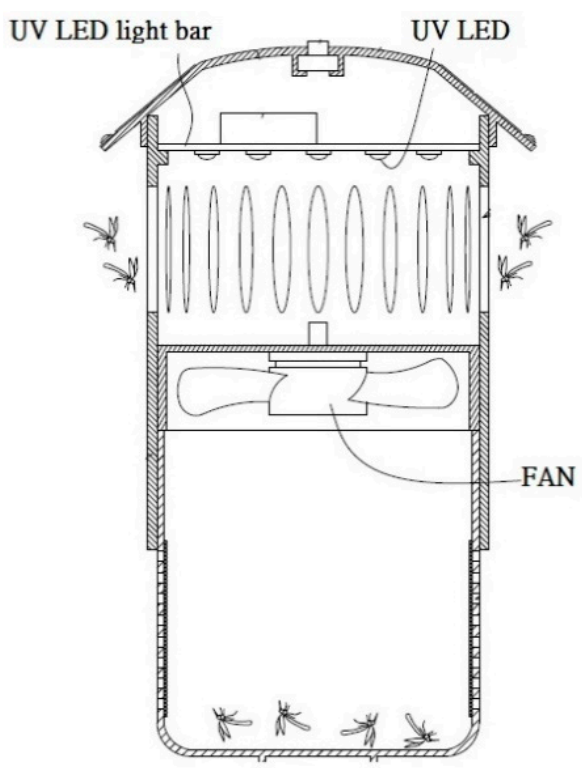

Figure 1. Conventional light-emitting diode (LED) mosquito trapping system.
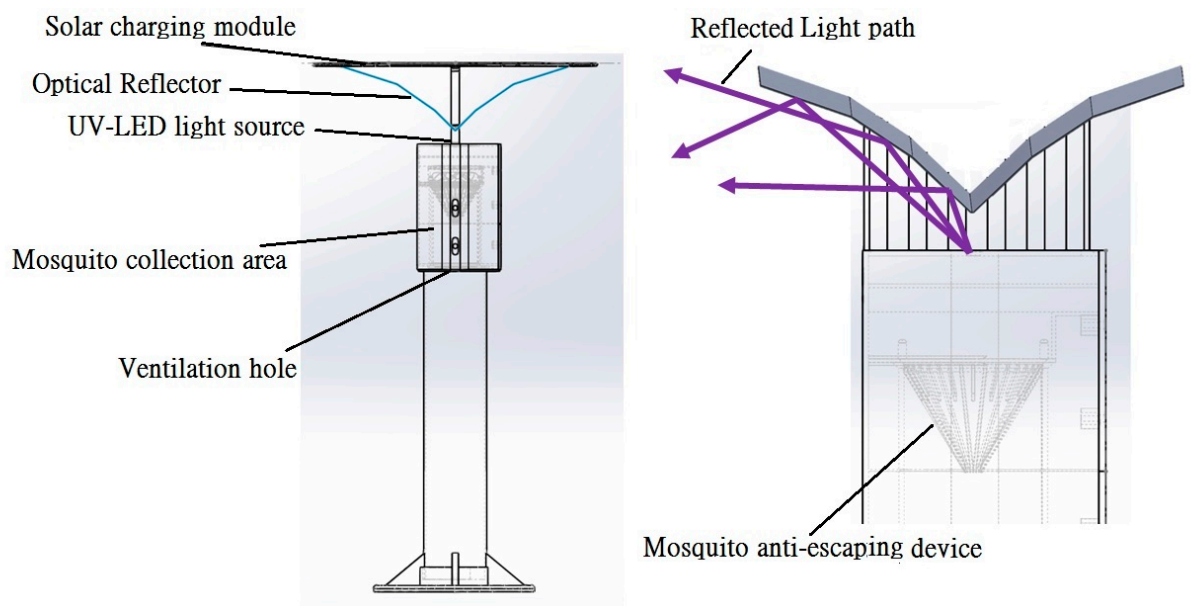

(a)

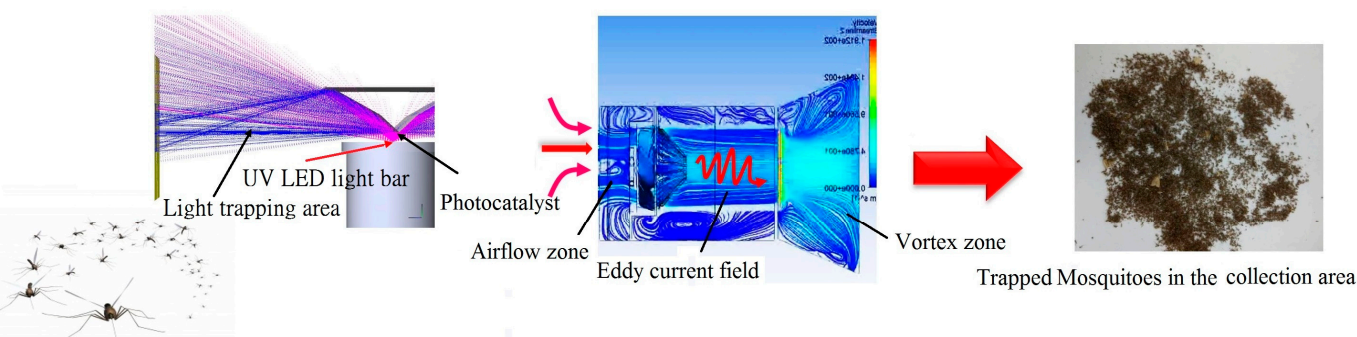

(b)

Figure 2. (a) Proposed mosquito trapping system; (b) Workflow of proposed mosquito trapping system. 


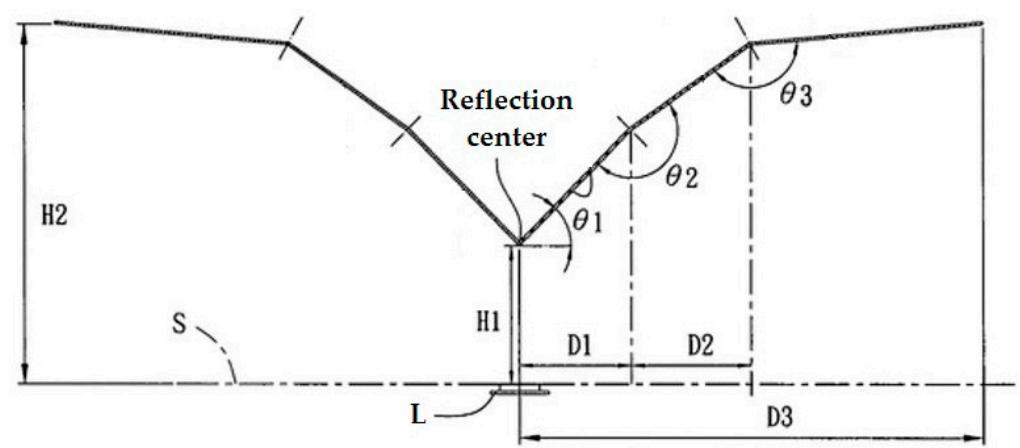

Figure 3. Mechanical drawing of the used reflector in the proposed system and its characteristic parameters.

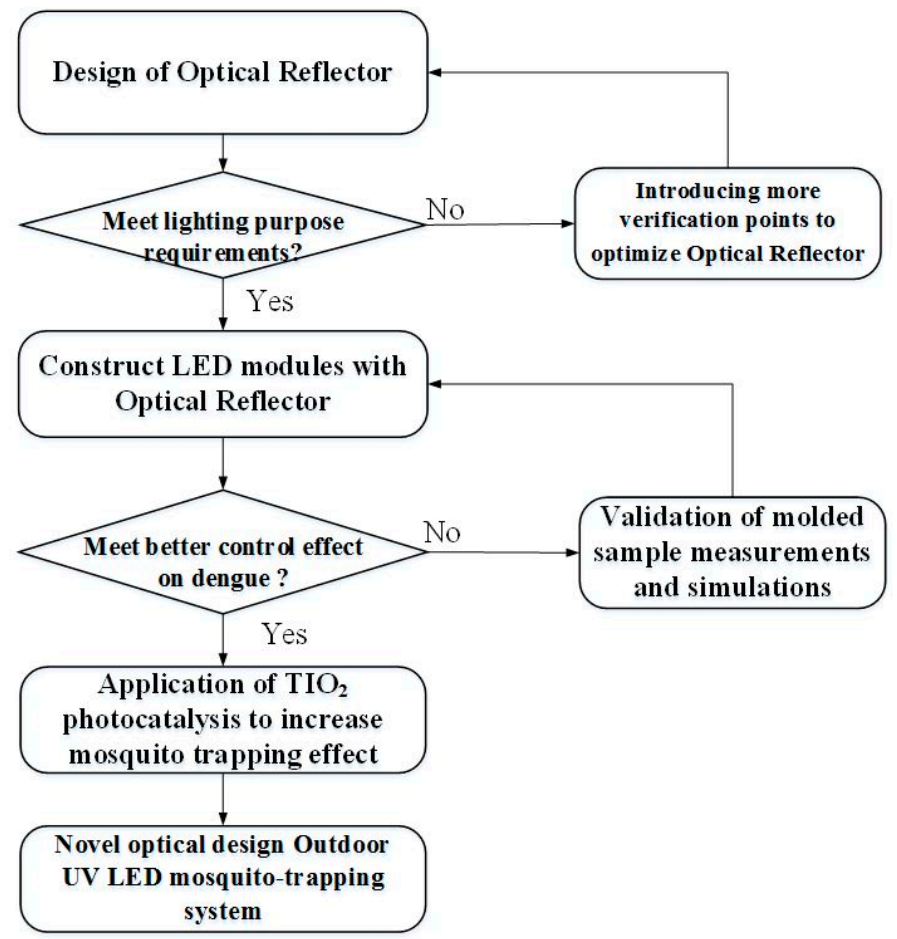

Figure 4. Workflow of optical-reflector design.

Most of the photocatalyst is made of semiconducting material, having an energy-band structure, as shown in Figure 5a. When excited by light, their valence electrons are in the lower energy-valence band, where electrons are trapped around atoms and are not active [14]. The pair of electron holes are excited by the photocatalyst after being illuminated, and nearby molecules $\left(\mathrm{H}_{2} \mathrm{O}, \mathrm{O}_{2}^{-}\right)$are ionized to form positive or negative ions, or free radicals $\left(\mathrm{OH}-, \mathrm{OH}^{*}\right)$.

$$
\begin{gathered}
\mathrm{TiO}_{2}+\mathrm{hv} \rightarrow \mathrm{h}^{+}+\mathrm{e}^{-} \\
\mathrm{OH}^{-}+\mathrm{h}^{+} \rightarrow \mathrm{OH}^{*} \\
\mathrm{O}_{2 \text { ads }}+\mathrm{e}^{-} \rightarrow \mathrm{O}_{2}{ }^{-} \text {ads }
\end{gathered}
$$

$\mathrm{O}_{2}{ }^{-}, \mathrm{H}+, \mathrm{OH}^{-}, \mathrm{OH}^{*}$, and $\mathrm{HO}_{2}$. plasmas and free radicals are produced $\mathrm{O}_{2}{ }^{-}$and $\mathrm{HO}_{2} \cdot$ radicals have a strong reducing ability, and $\mathrm{OH}^{-}$and $\mathrm{OH}^{*}$ radicals have a strong oxidizing ability, which can decompose other organic compounds on the surface of the photocatalyst into water and carbon dioxide [15].

$$
\mathrm{OH}^{*}+\text { pollutant }+\mathrm{O}_{2} \rightarrow \text { products }\left(\mathrm{CO}_{2}, \mathrm{H}_{2} \mathrm{O}, \ldots\right)
$$


I the stimuli of these valence electrons are given enough energy to overcome the energy gap and transition to the conduction band, however, they become active free electrons (represented by e-). At this time, the valence band leaves due to the electrons, relatively producing a positively charged active hole (indicated by $\mathrm{h}+$ ). Free electrons and holes are generated in pairs, so the number is the same. The electrons and holes generated by the photocatalyst can move freely. They may recombine to release energy, or they may move to the surface of the photocatalyst to decompose other organic compounds into water and $\mathrm{CO}_{2}[16]$, as shown in Figure 5b.

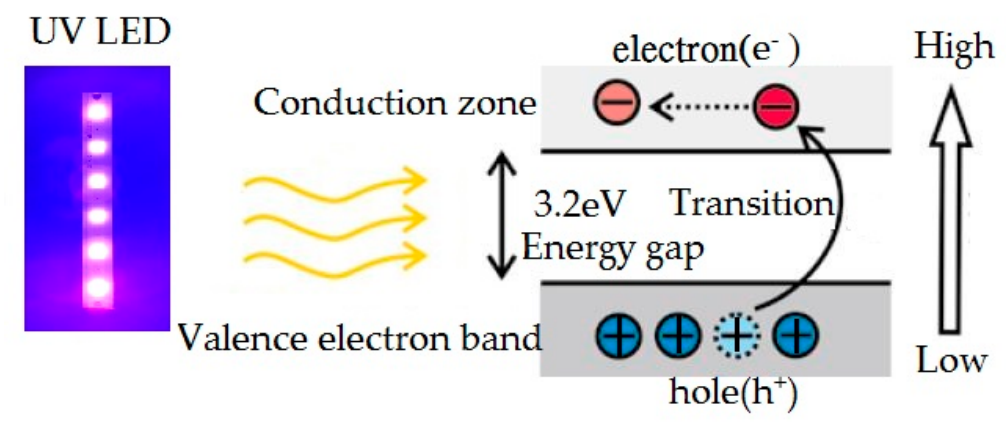

(a)

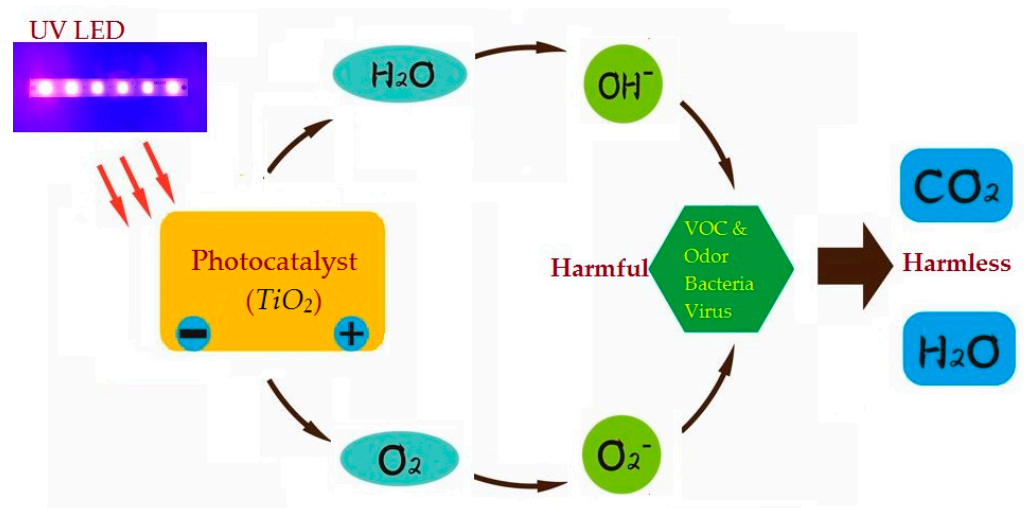

(b)

Figure 5. (a) $\mathrm{TiO}_{2}$ photocatalyst energy-band structure; (b) $\mathrm{TiO}_{2}$ photocatalyst $\mathrm{CO}_{2}$-producing reaction mechanism.

\section{Experiments and Results}

The UV LED strip light shown in Figure 6 has six chips, and each chip was derived in $0.5 \mathrm{~W}$ to emit UV light with a wavelength of $395 \mathrm{~nm}$, packaged in epoxy n: 1.5, to act as the source of the proposed system in the following experiments. The light distribution curve of the strip light was measured by ProMetric near-field measurement system (PM-NFMS), developed by Radiant Vision Systems Co., shown in Figure 6.
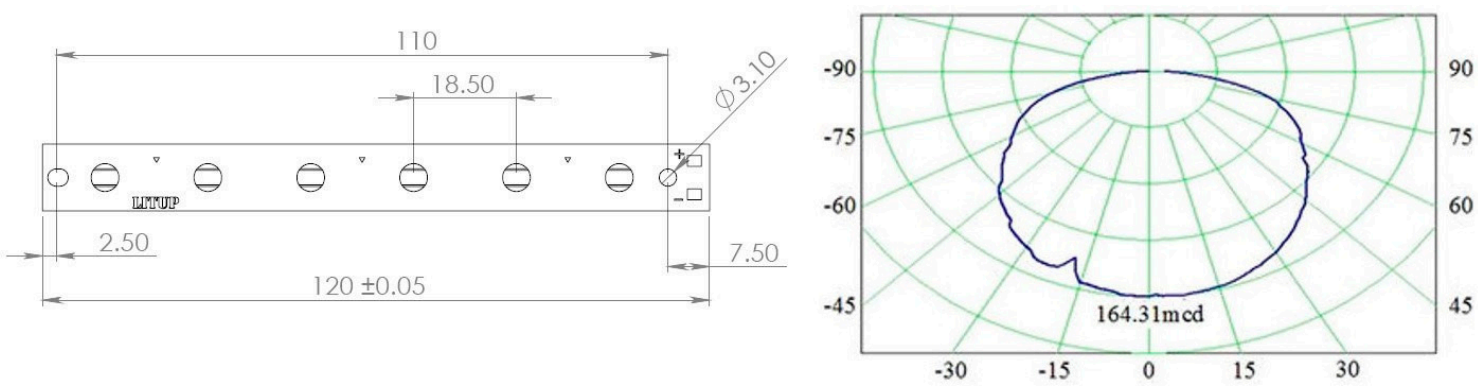

Figure 6. Ultraviolet (UV) LED light source used in the proposed system and its spatial light-intensity distribution curve. 
In the reflector-design procedure, the Di and $\theta_{\mathrm{i}}$ of the bird-wing reflector shown in Figure 3 were used as the variables to accomplish the targeted reflected light distribution. The light is located under the center of the reflector, about $50 \mathrm{~mm}\left(\mathrm{H}_{1}\right)$. The targets of the light-intensity distribution in optics design include Peak angle: $160^{\circ} \pm 5^{\circ}$ and Divergent angle referring the peak angle: $\leq 30^{\circ}$. For rapidly finding solutions in the optimization process, $\theta_{1}$ and $\theta_{2}$ were set as constants of 45 and 170 degrees, respectively. After optimization through SolidWorks, an optics design software with optimization functions, three different reflectors and their light distribution curves were prepared, as shown in Figure 7. The parameters of the optimized reflectors are listed in Figure 8.
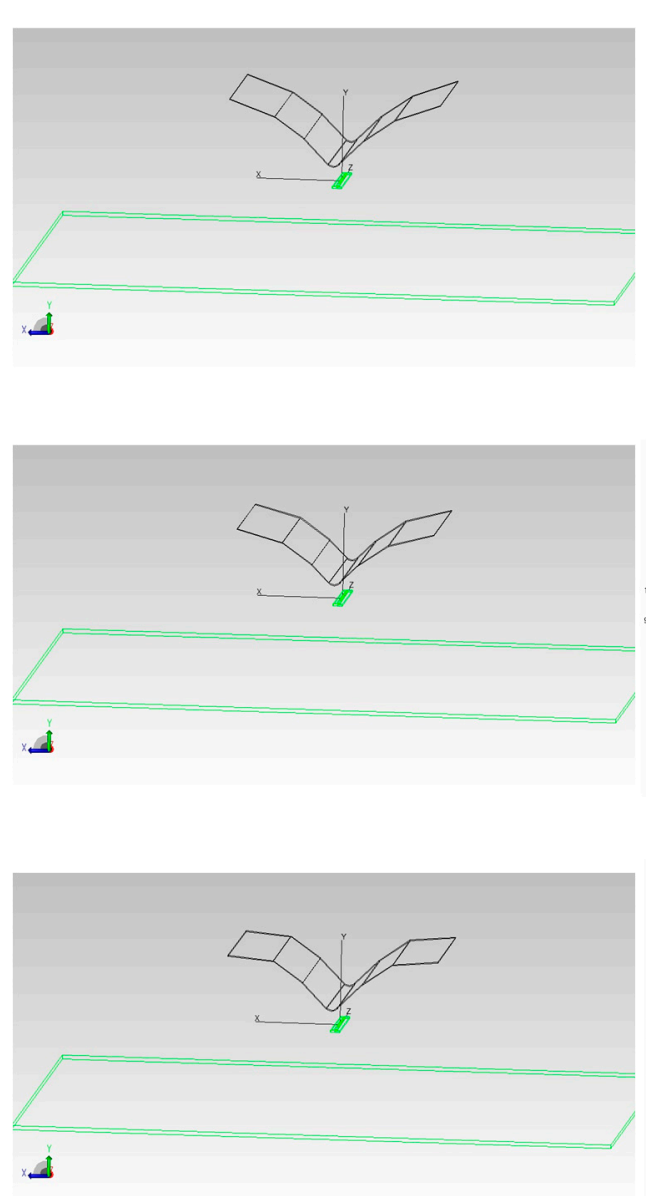

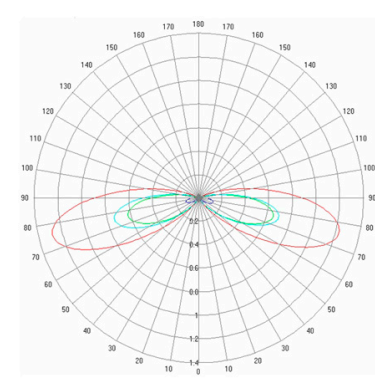

(a)

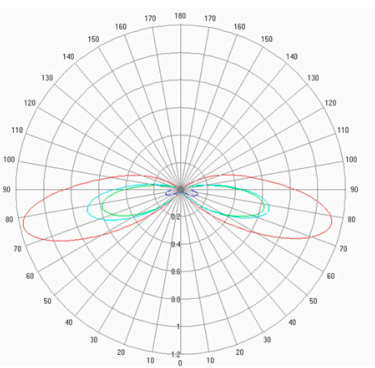

(b)

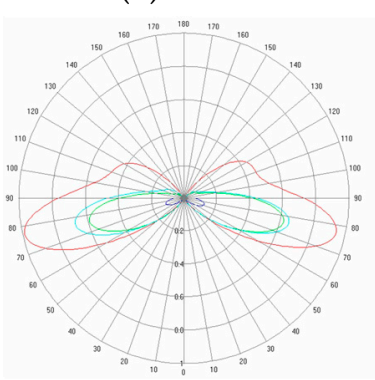

(c)
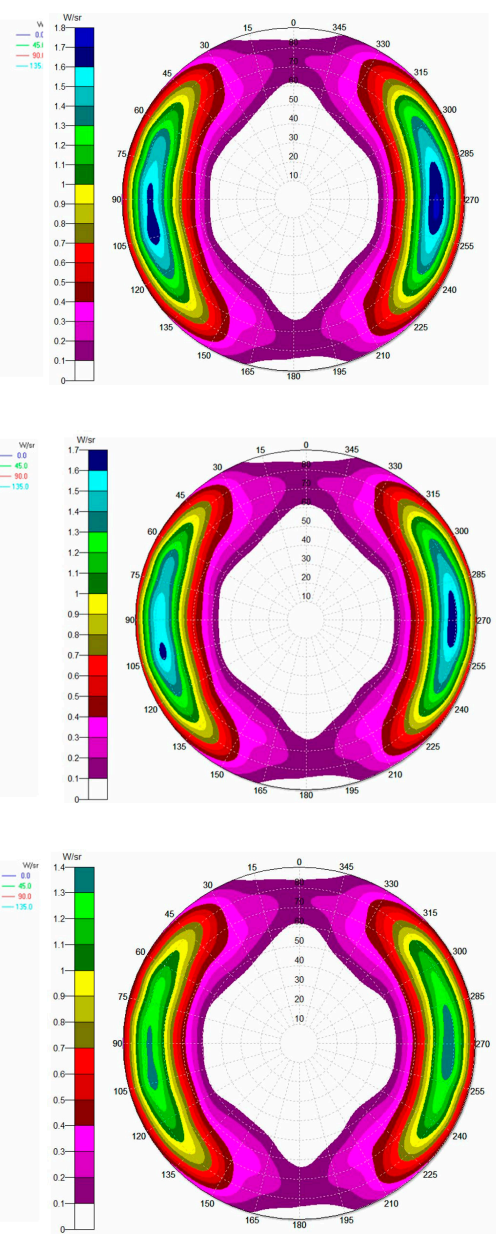

Figure 7. (a) Light distribution curves of the optimized type A reflector targeted at a 170 peak emitting angle; (b) Light distribution curves of the optimized type B reflector targeted at a 170 peak emitting angle; (c) Light distribution curves of the optimized type $C$ reflector targeted at a 170 peak emitting angle.

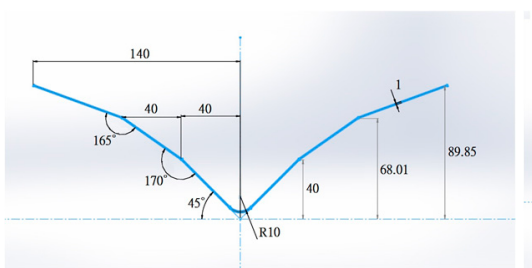

Type A

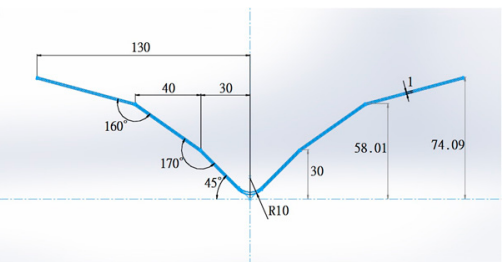

Type B

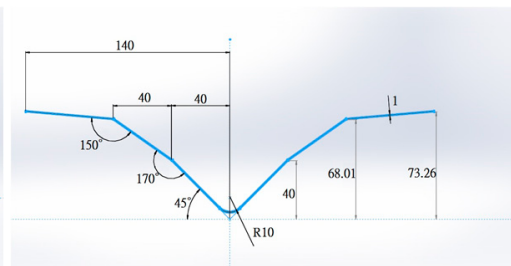

Type C

Figure 8. Optimized optical reflectors and their parameters. 
Type A: Angle $\theta_{1}$ is 45 degrees, angle $\theta_{2}$ is 170 degrees, angle $\theta_{3}$ is 165 degrees, projection distance $\mathrm{D}_{1}$ is $40 \mathrm{~mm}$, projection distance $\mathrm{D}_{2}$ is $40 \mathrm{~mm}$, and projection distance $\mathrm{D}_{3}$ is $140 \mathrm{~mm}$.

Type B: Angle $\theta_{1}$ is 45 degrees, angle $\theta_{2}$ is 170 degrees, angle $\theta_{3}$ is 160 degrees, projection distance $\mathrm{D}_{1}$ is $40 \mathrm{~mm}$, projection distance $\mathrm{D}_{2}$ is $40 \mathrm{~mm}$, and projection distance $\mathrm{D}_{3}$ is $130 \mathrm{~mm}$.

Type C: Angle $\theta_{1}$ is 45 degrees, angle $\theta_{2}$ is 170 degrees, angle $\theta_{3}$ is 150 degrees, projection distance $\mathrm{D}_{1}$ is $40 \mathrm{~mm}$, projection distance $\mathrm{D}_{2}$ is $40 \mathrm{~mm}$, and projection distance $\mathrm{D}_{3}$ is $140 \mathrm{~mm}$.

Based on the three different designs above, three sets of bird-wing reflectors were prototyped and installed with the airflow inhalation, respectively, for the experiments, as shown in Figure 9. A 3-W commercial mosquito trapper was also used in the experiments for comparison, as shown in Figure 10. After the experiments, the amount of captured mosquitoes for each testing day by the commercial trapper and the bird-wing trapper were all shown in Figure 11. Based on the results, the number of trapped mosquitos can be calculated such that the trapped mosquito numbers were up by $450 \%$ $((526 / 116) \times 100 \%)$ by using the proposed system with Type C reflector when compared with the conventional system. It can be found that Type $C$ was the best of all the experimental systems.
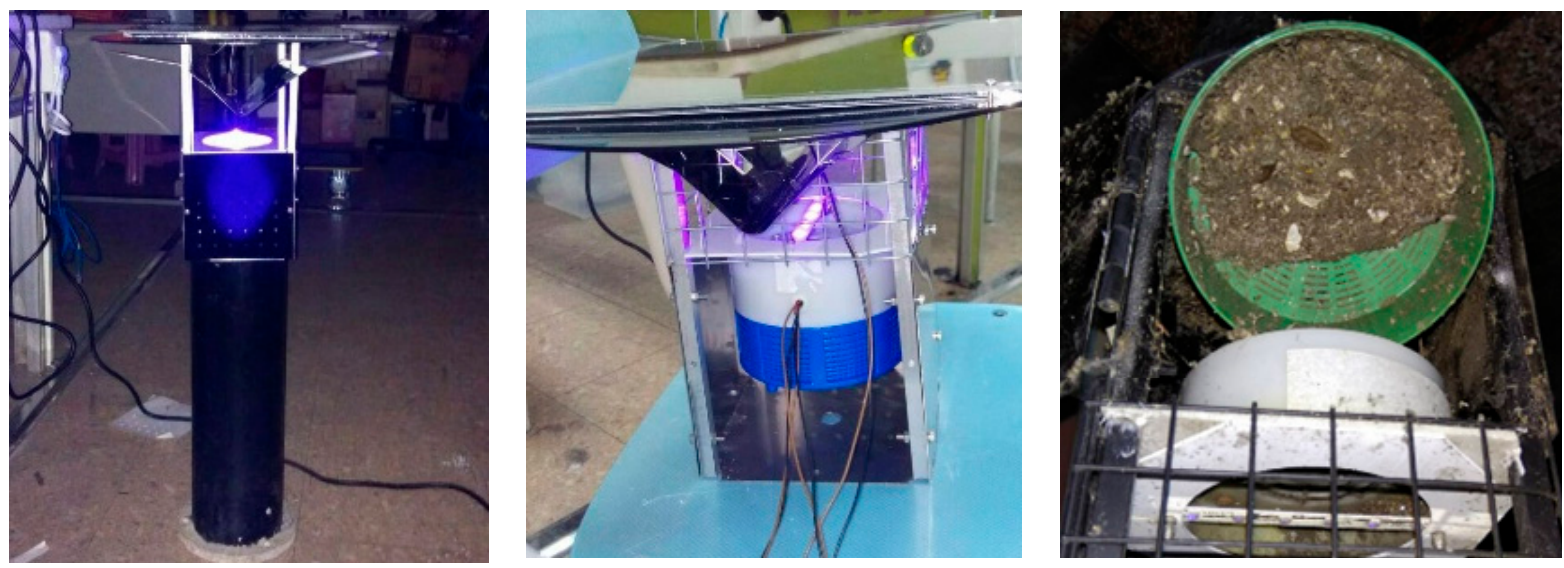

Figure 9. Proposed mosquito trapping system with a bird-wing optical reflector and its mosquito trapping results.
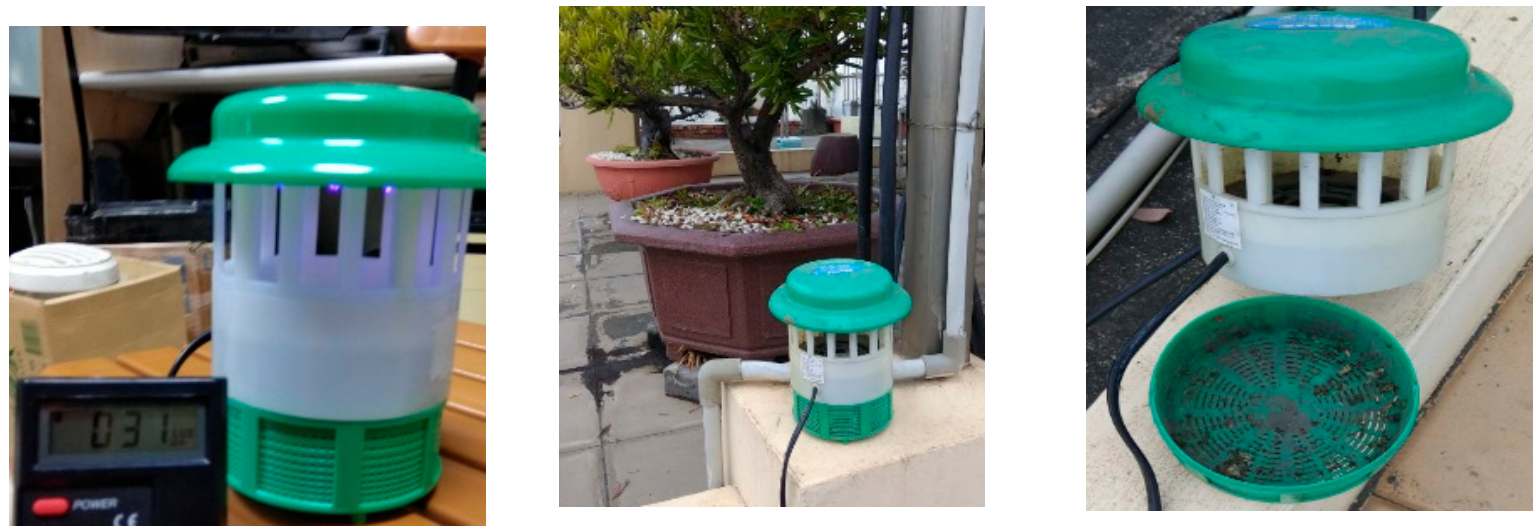

Figure 10. Conventional mosquito trapper and its mosquito trapping results.

In order to verify the effect of the photocatalytic coating on the proposed system, a $\mathrm{TiO}_{2}$ gel was applied on the center portion of the bird-wing optical reflector, as shown in Figure 12. The $\mathrm{TiO}_{2}$ gel was prepared by fully mixing $80 \% \mathrm{TiO}_{2}$ powder with $20 \%$ clean water in a beaker. Through a $\mathrm{CO}_{2}$-detecting instrument, $\mathrm{CO}_{2}$ concentration was measured besides, below, and away from the bird-wing reflector, respectively, as shown in Figure 13. The data were recoded for $12 \mathrm{~h}$ and are shown in Figure 13, which demonstrates that photocatalytic coating can really generate more $\mathrm{CO}_{2}$ around the system. 


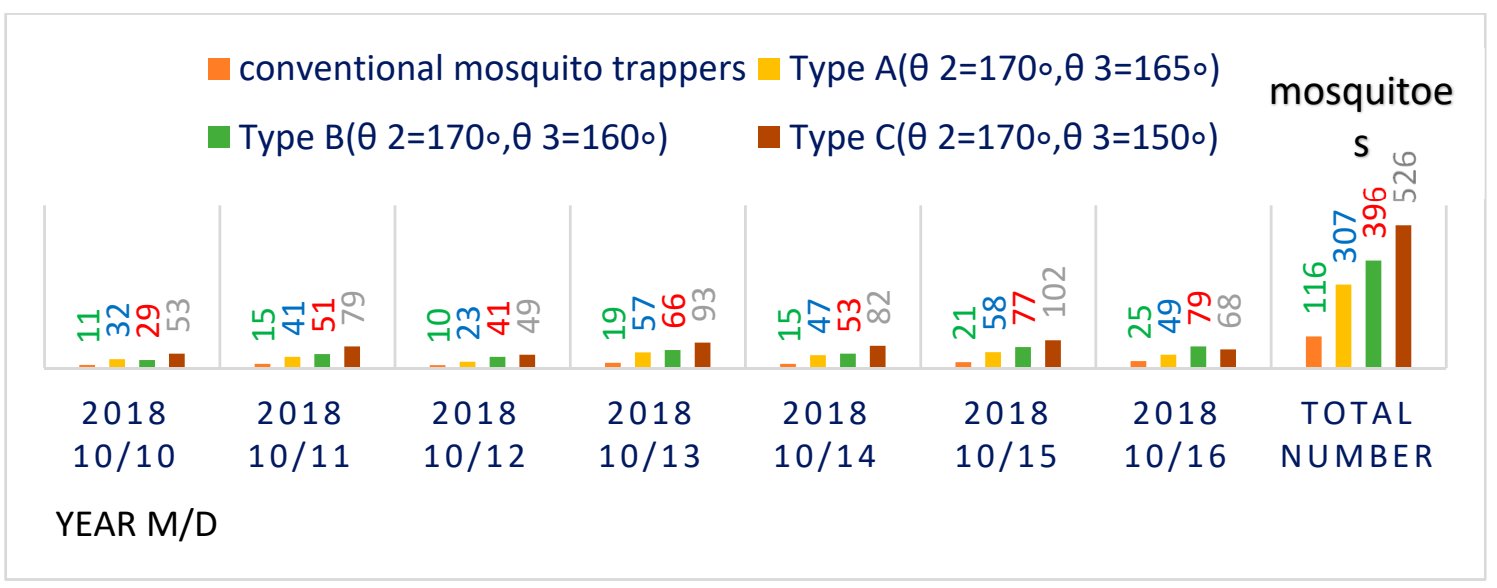

Figure 11. Comparison of proposed mosquito trapper with three different optimized reflectors and the conventional trapper with regard to mosquito trapping numbers.

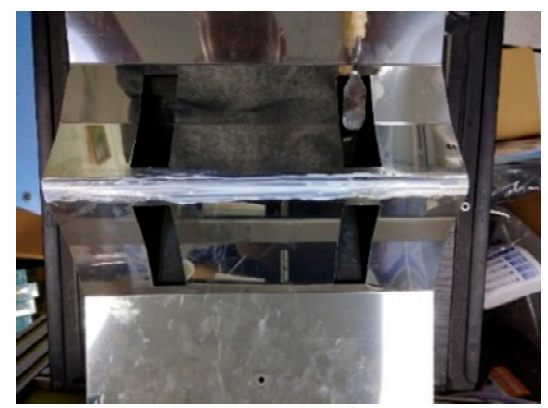

Figure 12. Coated $\mathrm{TiO}_{2}$ on bird-wing optical reflector.
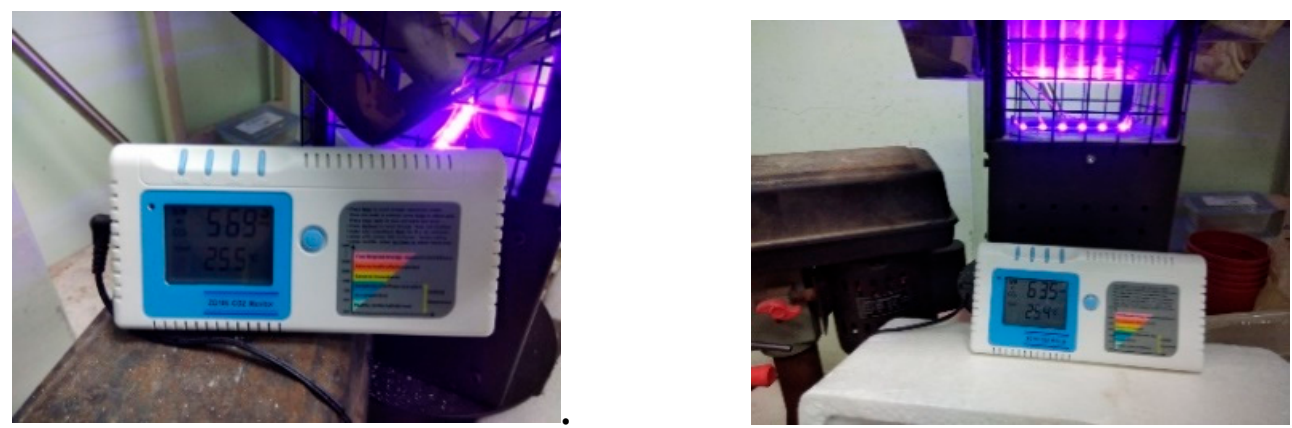

Figure 13. Detection and recording of $\mathrm{CO}_{2}$ concentration.

When light meets $\mathrm{TiO}_{2}$, it becomes a photocatalyst. $\mathrm{CO}_{2}$ is generated by the photocatalyst process. Insects know where a human being is by detecting the $\mathrm{CO}_{2}$ of people exhaling. Insect-killing devices imitate the respiration of human beings. $\mathrm{TiO}_{2}$ photocatalysts detect and record changes in $\mathrm{CO}_{2}$ concentration with a $\mathrm{CO}_{2}$ detection device $(12 \mathrm{~h})$, as shown in Figure 14.

Since $\mathrm{TiO}_{2}$ application is effective for $\mathrm{CO}_{2}$ production that mimics human exhalation, the proposed system with Type $\mathrm{C}$ reflector was used as the testing trapper to evaluate the difference before and after coating $\mathrm{TiO}_{2}$ on Type $\mathrm{C}$ reflector in mosquito trapping efficiency. After the experiments, the amount of captured mosquitoes for each testing day by the Type $\mathrm{C}$ bird-wing trapper and that with $\mathrm{TiO}_{2}$ coating were all shown in Figure 11. It can be figured out that the trapped amount could be further elevated by $45 \%((771-556) / 556 \times 100 \%)$ after applying the coating, as shown in Figure 15 . 


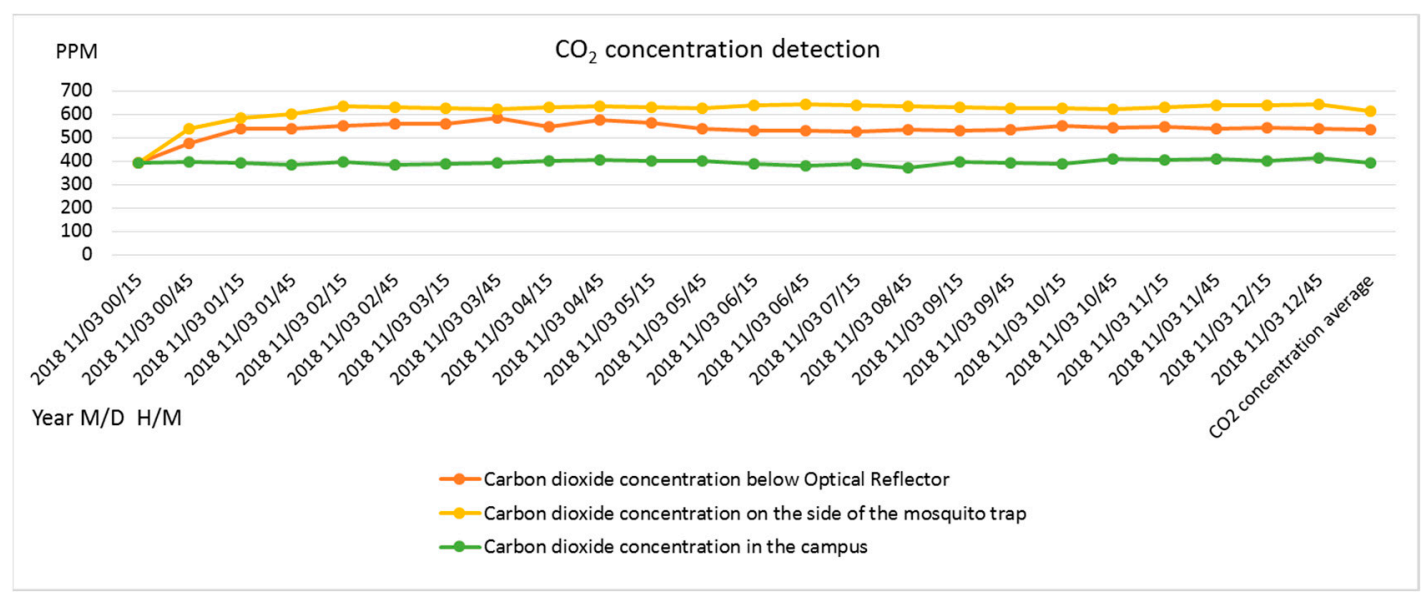

Figure 14. $\mathrm{CO}_{2}$ concentration besides, below, and away from the bird-wing reflector measured by the $\mathrm{CO}_{2}$ detection device for $12 \mathrm{~h}$, respectively.

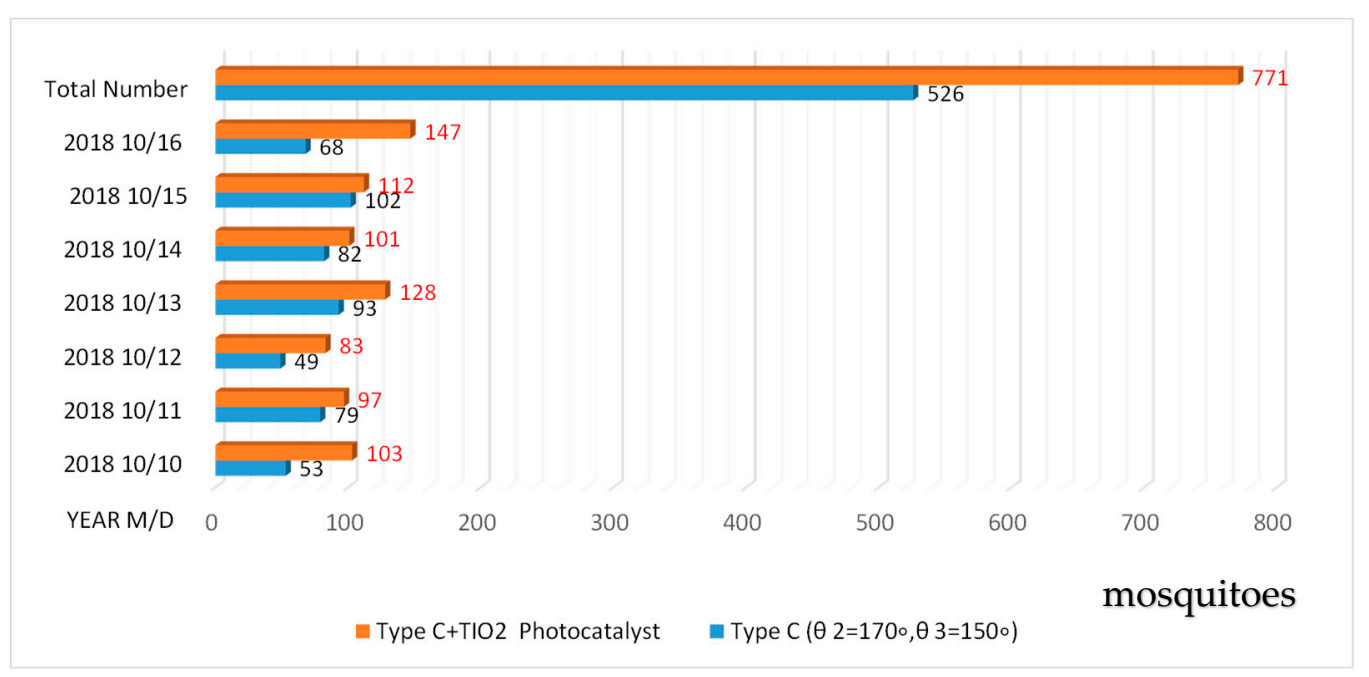

Figure 15. Comparison of mosquito trapping ability between the Type (c) mosquito trapping system with the bird-wing optical reflector, and that coated with $\mathrm{TiO}_{2}$.

\section{Discussion and Conclusions}

Dengue fever is the most serious vector-borne disease in Taiwan-there is still no vaccine or therapeutic drug. At present, the prevention and control strategy of dengue fever is still based on the principle of eliminating the source of vector mosquitoes and spraying chemical agents to kill vector-mosquito larvae. Although this method can quickly kill vector mosquitoes, they gradually gradually develop resistance. Modern technologies are dedicated to the development of chemicals that eliminate pests such as mosquitoes. After a long period of time, organism resistance becomes stronger, and the high concentration of pesticides directly or indirectly harming humans through the food chain is becoming increasingly serious.

This study is dedicated to the development of a new mosquito trapper that target the physical trapping factors of mosquitos including lighting, color and evaluation tests in parks, primary schools and hospitals of southen Taiwan. Currently, LEDs with $395 \mathrm{~nm}$ or $365 \mathrm{~nm}$ are both the mainstream products in the UV LED market. But, so far, $365 \mathrm{~nm}$ LED is still more expensive and much less efficient than a $395 \mathrm{~nm}$ LED. In consequence, $395 \mathrm{~nm}$ LED is adopted as the source in the experiments instead of $365 \mathrm{~nm}$ LED, although $365 \mathrm{~nm}$ is usually the UV peak wavelength of conventional mosquito trapper using fluorescent light tubes. The light-intensity distribution peak angle and the divergent angle centering the peak angle of the new trapper are targeted as $160^{\circ} \pm 5^{\circ}$ and $\leq 30^{\circ}$, respectively in 
the reflector optimization process. The $160^{\circ}$ peak angle is for covering the trapping area as far as possible and the limited $30^{\circ}$ divergent angle is for restricting upward UV rays generation to decrease the glare to human eye. The study's experiment results show that the equal-intensity curve (red line) with a radiation intensity of $135 \mathrm{w} / \mathrm{sr}$ has a lightlike curve, similar to the shape of a butterfly wing, indicating that the range of light divergence is wider. This study demonstrates that the optimization of a bird-wing optical reflector for outdoor UV LED mosquito systems can better attract mosquitoes (as shown in Figures 7c and 11). Compared with a conventional mosquito trapping device (shown in Figure 1), the outdoor trapping range can be extended to $225 \pi \mathrm{m}^{2}$, and the number of captured mosquitoes increased by about $450 \%$.

In addition, applying $\mathrm{TiO}_{2}$ to the bird-wing optical reflector of the UV LED mosquito trapping system can not only imitate human breath, but also attract mosquitoes to enter the mosquito trapping system. Compared with the anti-mosquito effect of each group of bird-wing optical reflectors of the UV LED mosquito-removing system, it can increase mosquito-killing efficiency by more than $45 \%$.

The UV LED mosquito trapping system with a photocatalyst optical reflector operates for about $24 \mathrm{~h}$. It begins to catch mosquitoes immediately when there is activity in the area. Female mosquitoes looking for blood are drawn to the trapping device and eliminated. The trap gradually reduces the number of mosquitoes as it attracts and destroys them. It may take 6-8 weeks of use to reduce the number of mosquitoes to the point where the proposed device has successfully interrupted the mosquito breeding cycle [17].

Therefore, the bird-wing optical reflector with a photocatalyst for a low-glare mosquito trapping system with light-emitting diodes has been scientifically proven to be safe and effective in reducing the number of mosquitoes, and to be the best auxiliary solution for dengue fever.

Author Contributions: Conceptualization, W.-H.T., Y.C.C., C.-H.C. and H.-Y.L.; Data curation, W.-H.T. and C.-H.C.; Formal analysis, W.-H.T. and H.-Y.L.; Funding acquisition, D.J., W.-C.H. and H.-Y.L.; Methodology, W.-H.T. and Y.C.C.; Project administration, W.-C.H. and H.-Y.M.; Resources, D.J., W.-C.H., Y.C.C., C.-H.C., H.-Y.M. and H.-Y.L.; Supervision, D.J. and H.-Y.M.; Validation, W.-H.T.; Visualization, C.-H.C. and H.-Y.L.; Writing-Original Draft, W.-H.T.; Writing-Review \& Editing, H.-Y.L. The authors of the present work made contributions to all of its parts.

Funding: This research received funding from Yuan General Hospital. This work was supported by the National Science Council of the Republic of China, project MOST 107-2622-E-992-002-CC3.

Conflicts of Interest: The authors declare no conflict of interest.

\section{References}

1. WHO. Dengue and Severe Dengue. Available online: http://www.who.int/topics/dengue/en/ (accessed on 12 January 2015).

2. Zinser, M.; Ramberg, F.; Willott, E. Scientific Note Culex quinquefasciatus (Diptera: Culicidae) as a potential West Nile virus vector in Tucson, Arizona: Blood meal analysis indicates feeding on both humans and birds. J. Insect Sci. 2004, 4, 20. [CrossRef] [PubMed]

3. Chang, C.; Ortiz, K.; Ansari, A.; Gershwin, M.E. The Zika outbreak of the 21st century. J. Autoimmun. 2016, 68, 1-13. [CrossRef] [PubMed]

4. Chen, H.L.; Tang, R.B. Why Zika virous infection has become a public health concern. J. Chin. Med. Assoc. 2016, 79, 174-178. [CrossRef] [PubMed]

5. Centers for Disease Control, Ministry of Health and Welfare, R.O.C. (Taiwan). An Introduction to the Epidemiology of Dengue Fever, Prevention Policies, and Relevant Laws and Regulations. Available online: http:/ / www.cdc.gov.tw / downloadfile.aspx?fid=00A3CFA-4265F1A4C (accessed on 5 January 2015).

6. Bidlingmayer, W.L. How mosquitoes see traps: Role of visual responses. J. Am. Mosq. Control Assoc. Mosq. News 1994, 10, 272-279.

7. Browne, S.M.; Bennett, G.F. Response of mosquitoes (Diptera: Culicidae) to visual stimuli. J. Med. Entomol. 1981, 18, 505-521. [CrossRef] [PubMed]

8. Kay, R.E. Fluorescent materials in insect eyes and their possible relationship to ultra-violet sensitivity. J. Insect Physiol. 1969, 15, 2021-2028. [CrossRef] 
9. Shimoda, M.; Honda, K.I. Insect reactions to light and its applications to pest management. Appl. Entomol. Zool. 2013, 48, 413-421. [CrossRef]

10. Burkett, D.A.; Butler, J.F.; Kline, D.L. Field evaluation of colored light-emitting diodes as attractants for woodland mosquitoes and other Diptera in north central Florida. J. Am. Mosq. Control Assoc. Mosq. News 1998, 14, 186-195.

11. Adriana, D.B.; Lars, C. The Evolution of Color Vision in Insects. Annu. Rev. Entomol. 2001, 46, 471-510.

12. Muir, L.E.; Kay, B.H.; Thorne, M.J. Aedes aegypti (Diptera: Culicidae) Vision: Response to Stimuli from the Optical Environmen. J. Med. Entomol. 1992, 29, 445-450. [CrossRef] [PubMed]

13. Burkett, D.A.; Butler, J.F. Laboratory evaluation of colored light as an attractant for female Aedes aegypti, Aedes albopictus, Anopheles quadrimaculatus, and Culex nigripalpus. Fla. Entomol. 2005, 88, 383-389. [CrossRef]

14. Fujishima, A.; Rao, T.N.; Tryk, D.A. $\mathrm{TiO}_{2}$ Photocatalysis. J. Photochem. Photobiol. C Photochem. Rev. 2000, 1, 1-21. [CrossRef]

15. Hiroshi, T. Photocatalyst Diagram; Shangzhou Publishing: Taipei, Taiwan, 2003.

16. Hashimoto, F.S.; Ren, W.J. Graphic Photocatalyst; Publishing Co., Ltd: Taipei, Taiwan, 2006.

17. Introduction to the Ecology and Habits of Dengue Vector Mosquitoes; Department of Disease Control of the Department of Health: Taipei, Taiwan, 2014.

(C) 2019 by the authors. Licensee MDPI, Basel, Switzerland. This article is an open access article distributed under the terms and conditions of the Creative Commons Attribution (CC BY) license (http://creativecommons.org/licenses/by/4.0/). 